Radiologe 2009 · 49:425-433

DOI 10.1007/s00117-009-1833-z

Online publiziert: 18. Mai 2009

(c) Springer Medizin Verlag 2009

T.C. Mamisch', 2 S. Werlen ${ }^{2} \cdot$ C. Zilkens ${ }^{3} \cdot$ S. Trattnig ${ }^{2}$-Y.-J. Kim ${ }^{4} \cdot$ K.A. Siebenrock ${ }^{1}$. B. Bittersohl ${ }^{1}$

${ }^{1}$ Abt. Orthopädische Chirurgie, Inselspital, Bern

${ }^{2}$ Abt. Radiologie, Klinik Sonnenhof, Bern

${ }^{3}$ Orthopädische Klinik, Universitätsklinikum Düsseldorf, Düsseldorf

${ }^{4}$ Childrens Hospital, Harvard Medical School, Boston

\title{
Radiologische Diagnose des femoroazetabulären Impingements
}

\begin{abstract}
Bei der Hüftgelenkdysplasie besteht eine insuffiziente Überdachung des Femurkopfs [1]. Dies führt zu einer erhöhten lateralen Druckbelastung [2] und im weiteren Verlauf zu einer arthrotischen Destruktion des Gelenks [3]. Im Gegensatz dazu ist der Pathomechanismus beim femoroazetabulären Impingement (FAI) komplexer. Hier bewirkt ein anatomisches Missverhältnis zwischen proximalem Femur (Übergang Femurkopf/Schenkelhals) und Azetabulum eine frühzeitige Abnützung der Gelenkflächen [4]. Unter Impingement versteht man in diesem Fall, ähnlich dem Impingement der Schulter, einen pathologischen mechanischen Kontakt zweier artikulierender Gelenkteile.
\end{abstract}

\section{Hintergrund}

Erstmals wurde das FAI 1991 von Ganz et al. [5] als Komplikation nach Schenkelhalsfrakturen beschrieben. Die Beziehung zwischen diesen Komplikationen und degenerativen Knorpelveränderungen in der Hüfte wurde zu diesem Zeitpunkt jedoch noch nicht erkannt. Die radiologische Anomalität in Form einer Femurkopf-HalsÜbergangsstörung, die zu einer Hüftgelenkarthrose bei jungen Patienten führte, wurde schon zuvor beschrieben, z. B. als" Pistolengriffdeformität” bei Patienten mit einer Ephihyseolysis capitis femoris [6]. Es bleibt jedoch unklar, ob und in wel- chem Ausmaß milde Formen der Ephihyseolysis capitis femoris zur Entstehung einer vorzeitigen Hüftgelenkarthrose führen und ob die "präarthrotische Deformität” [7], die“Tiltdeformität” [8] und die“P istolengriffdeformität” [6] Auslöser oder Folge der Degeneration sind. Myers et al. [9] konnten 1999 das FAI als Ursache für Hüftschmerzen und Hüftgelenkdegeneration bei Dysplasiepatienten, bei denen initial zur Verbesserung der Minderüberdachung des Femurkopfs eine periazetabuläre Osteotomie (PAO) durchführt worden war, nachweisen ("Bernese disease"). Das Impingement der artikulären Gelenkflächen aufgrund morphologischer Anomalitäten von proximalem Femur und/ oder Azetabulum wurde hierbei intraoperativ bestätigt.

In weiteren Studien konnte das FAI als Ursache einer Hüftgelenkarthrose bestätigt werden $[10,11,12,13,14,15,16]$.

\section{Formen des FAI}

Anhand des Pathomechanismus werden 2 Formen des FAI unterschieden [4]:

- Cam-Impingement und

- Pincer-Impingement.

Die Klassifizierung erfolgt nach klinischen und röntgenradiologischen Parametern.

Das Cam-(Nockenwellen-)Impingement ist die femorale Form des FAI. Hierbei führt ein entrundeter Hüftkopf zur Verminderung oder Aufhebung der
Taillierung („offset“) zwischen Kopf und Schenkelhals. Dieser Bereich, die so genannte Bumpdeformität, dringt bei Flexion und Innenrotation in das Gelenk ein und führt zu pathologischen Scherkräften und schließlich zu Labrum- und Knorpelschäden [10, 11, 13]. Diese Anomalität im Bereich des femoralen Kopf-Hals-Übergangs, die häufig im anteriosuperioren Bereich zu finden ist [17], resultiert aus anatomischen Veränderungen, verursacht durch Schenkelhalsfrakturen [18], Ephihyseolysis capitis femoris [19, 20] und Morbus-Legg-Calvé-Perthes-Fehlstellungen $[21,22]$. Ein Cam-Impingement ist häufig bei jungen Männern im Alter von 20-30 Jahren anzutreffen.

Das Pincer- (Beißzangen-)Impingement ist die azetabuläre Form des FAI und resultiert aus einer generalisierten oder lokalen Mehrüberdachung des Femurkopfs $[4,23]$. Eine generalisierte Mehrüberdachung ist häufig assoziiert mit einer erhöhten Tiefe der azetabulären Fossa, wie sie bei der Coxa profunda und der azetabulären Protrusion gefunden wird. Eine lokale Mehrüberdachung findet sich häufig bei der azetabulären Retroversion (anteriore Mehrüberdachung $[14,15]$ ) sowie bei einer prominenten Azetabulumhinterwand (posteriore Mehrüberdachung [24]). Auch eine Überkorrektur durch Beckenumstellungsosteotomien kann einen Pincer-Effekt verursachen ("Bernese disease" [25]). Femurkopf und/oder Femurkopf-Hals-Übergang sind bei der 
reinen Pincer-Form morphologisch unverändert.

Beim Pincer-Impingement kommt es bei Flexion im Hüftgelenk zu einem frontalen Anschlagen des Femurhalses an das azetabuläre Labrum im anteriosuperioren Bereich. Dieses repetitive Anschlagen führt zur Labrumdegeneration, -verknöcherung, Zystenbildung im Labrum und schließlich zum Labrumabriss. [4] Der angrenzende Knorpel ist im Verlauf ebenfalls von dieser mechanischen Einwirkung betroffen. Morphologisch ist der Knorpelschaden zunächst auf einen schmalen Rand begrenzt [10]. Aus dem frontalen Anschlag des Femurs an den Azetabulumvorderrand resultiert zudem eine Hebelwirkung, die die Rückseite des Femurkopfs an die Azetabulumhinterwand luxiert. Dies führt, neben den Schäden im vorderen, auch zu degenerativen Veränderungen im hinteren Bereich $[4,10$, 26]. Diese so genannte Contre-coup-Läsion kommt bei ca. 1/3 der genannten Fälle vor. Ein Pincer-Impingement ist häufig bei Frauen im Alter um 40 Jahre anzutreffen.

Beide Impingementtypen kommen selten isoliert vor. Die meisten FAI-Patienten $(86 \%)$ zeigen röntgenradiologisch eine Kombination beider Impingementformen [10].

\section{Klinische Relevanz}

Typische Symptome sind eingeschränkte Flexion und Innenrotation sowie Schmerzen in der Leiste insbesondere nach längerem Sitzen oder nach sportlichen Aktivitäten [4]. Bei der klinischen Untersuchung zeigt sich ein reproduzierbarer Schmerz in der Leiste in $90^{\circ}$ Hüftflexion und forcierter Innenrotation (positiver vorderer Impingementtest $[5,27]$ ). Dieser Schmerz wird mit einer Läsion des azetabulären Labrums assoziiert. Zudem wird eine Verbindung dieser Schmerzsymptome mit der Dehnung der Gelenkkapsel und des Lig. capitis femoris diskutiert, was bisher noch nicht weiter untersucht wurde.

Es ist ausführlich dokumentiert, dass das FAI neben den genannten Symptomen häufig zu osteoarthrotischen Veränderungen im Hüftgelenk führt [4, 10, 11, $12,13,14,15,16,18,21,23,28,29]$ Um die
Symptome zu beheben (Einschränkung der Gelenkmobilität, Schmerzen) und um der weiteren Degeneration vorzubeugen, ist eine Operation angeraten [30]. Hierbei werden die Ursachen des Impingements mittels einer chirurgischen Hüftluxation $[4,31,32]$ oder einer Hüftarthroskopie bei wenig komplexen morphologischen Anomalitäten [33] beseitigt. Dies beinhaltet die Verbesserung des femoralen KopfSchaft-Offsets ("Bumpektomie") beim Cam-Impingement und/oder die azetabuläre Pfannenrandtrimmung mit anschließender Labrumrefixation beim PincerImpingement.

Bei einer azetabulären Retroversion kann auch eine antevertierende periazetabuläre Osteotomie (PAO) notwendig sein [15]. Die chirurgische Hüftluxation ist ein sicheres Verfahren, das eine genaue Beurteilung von Gelenkstrukturen wie Labrum und Knorpel ermöglicht. Mittelfristige Nachuntersuchungen zeigen gute Resultate bei Patienten mit präoperativ wenig fortgeschrittener Gelenkdegeneration $[21,28,34]$. Hingegen waren die klinischen Resultate bei präoperativ bereits fortgeschrittener Arthrose schlecht. Dies erklärt die Wichtigkeit einer sensitiven als auch akkuraten Diagnostik, um Frühstadien der Gelenkschädigung erkennen zu können (wovon die Patienten bei einer Operation am meisten profitieren können).

Die Diagnose "FAI" wird anhand klinischer und röntgenradiologischer Parameter gestellt. Die Magnetresonanztomographie (MRT) nativ oder mit Kontrastmittelapplikation (MR-Arthrographie [MRA]) wird eingesetzt, um Labrum-, Knorpel- und weitere Gelenkschäden erkennen und das Ausmaß der Schädigung beurteilen zu können.

Die radiologische Untersuchung des Hüftgelenks erfordert klinische Erfahrung und die technischen Voraussetzungen, um eine optimale Befunderhebung zu ermöglichen. Dies ist bedingt durch die räumliche Komplexität dieses Gelenks, wo sich die Gelenkflächen dreidimensional sphärisch gegenüberstehen und die Gelenkstrukturen wie Gelenkkapsel, Labrum und Knorpel eng beieinander liegen und somit Schäden der einen Struktur häufig mit Schäden der anderen Teile einhergehen. Demzufolge erfordert die radiolo- gische Beurteilung des Hüftgelenks einerseits eine genaue Untersuchung der einzelnen Strukturen und auch einen guten Überblick über das Gelenk im Ganzen.

\section{Ziel der Arbeit}

Die vorliegende Arbeit gibt eine Übersicht über radiologische Diagnoseverfahren beim FAI und erläutert Kriterien zur Diagnose und Klassifizierung der FAI-Formen. Standardmethoden, ihre Limitierungen und mögliche Lösungen und Verbesserungen werden diskutiert. Das beinhaltet röntgenradiologische und tomographische (MRT, MRA) Standardverfahren sowie als Ausblick technische Weiterentwicklungen in Form biochemisch sensitiver MRT-Verfahren, die eine noch genauere und auch vereinfachende Diagnostik im Hüftgelenk ermöglichen.

\section{Röntgenradiologische Kriterien}

Die native Röntgenuntersuchung ist das Standardverfahren zur primären $\mathrm{Be}$ funderhebung, FAI-Klassifikation und Beurteilung des Ausmaßes der morphologischen Veränderung. Wird hierbei ein FAI diagnostiziert, folgen eine MRT- oder MRA-Untersuchung, um das Ausmaß eventueller Schäden an Labrum, Knorpel und Bindegewebe genauer charakterisieren zu können.

Die Standardröntgenradiologische Untersuchung beim FAI beinhaltet 2 Röntgenaufnahmen [4]:

- eine a.p.-Beckenaufnahme sowie

- eine laterale „Cross-table”-Aufnahme des proximalen Femurs.

Die a.p.-Beckenaufnahme wird im Liegen und mit dem zu untersuchenden Bein in $15^{\circ}$ Innenrotationsstellung durchgeführt, um die femorale Antetorsion auszugleichen und die Konturen des lateralen Femurkopf-Schaft-Übergangs besser darstellen zu können [24]. Der Röntgenstrahlfokus wird dabei auf den Mittelpunkt zwischen beiden Spinae iliacae anteriores superiores auf Höhe des Symphysenoberrandes gerichtet. Die a.p.-Beckenaufnahme muss in Beckenneutralstellung erfolgen, da eine vermehrte Kipp- und Rotationsstellung zu einer falschen Betonung des Retroverionszeichens führt und 
umgedreht $[24,35,36,37]$. Als Richtlinie für eine neutrale Beckenkippstellung wurde ein Abstand von 3,2 cm zwischen Symphysenoberrand und Mittelpunkt des Sakrokokzygealgelenks bei Männern und $4,7 \mathrm{~cm}$ bei Frauen vorgeschlagen [35]. Eine genauere Aussage über die Kippstellung des Beckens kann mit einer Beckenseitaufnahme gemacht werden. Hierbei wird die Beckenstellung als neutral definiert, wenn der Winkel zwischen einer Horizontalen und der Linie, die Symphysenoberrand und Sakrumpromontorium verbindet, $60^{\circ}$ beträgt [24].

Der Femurkopf-Schaft-Übergang wird in der lateralen Cross-table-Aufnahme beurteilt [38]. Für diese Röntgenaufnahme wird der Röntgenfilm, lateral der zur untersuchenden Hüfte, parallel zum Schenkelhals angeordnet. Die Strahlenquelle ist medial der zu untersuchenden Seite und dabei so gerichtet, dass der Strahlenfokus über den Untersuchungstisch senkrecht durch den Schenkelhals verläuft und auf den lateral befindlichen Röntgenfilm trifft. Der Patient muss während dieser Aufnahme das Bein auf der Gegenseite flektieren und abduzieren, um somit den erforderlichen Raum für Strahlenquelle und -gang zu verschaffen.

\section{Typische röntgenradiologische Zeichen beim Cam-Impingement}

Die Aspherität des Femurkopfs bzw. die Verminderung oder Aufhebung der Taillierung (Offset) zwischen Kopf und Schenkelhals wird mittels $\alpha$-Winkel, anteriorem Offset und/oder Offsetratio in der Cross-table-Aufnahme quantifiziert [13]. Der $\alpha$-Winkel wird gebildet zwischen (• Abb. 1):

- der Linie, die das Hüftkopfzentrum mit dem Punkt, an dem der Hüftkopf-Schenkelhals-Übergang die sphärische Kontur des Kopfes verlässt, verbindet und - der femoralen Schenkelhalsachse.

Winkel größer als $50^{\circ}$ sind ein Hinweis für eine Anomalität im Femurkopf-Schenkelhals-Übergang. Das anteriore Offset ist definiert als Differenz zwischen anteriorem Femurkopfradius und dem anterioren Femurschaftradius am Femurkopf-Schenkelhals-Übergang. An asymp-

Radiologe 2009 · 49:425-433 DOI 10.1007/s00117-009-1833-z

(C) Springer Medizin Verlag 2009

T.C. Mamisch · S. Werlen · C. Zilkens · S. Trattnig · Y.-J. Kim · K.A. Siebenrock · B. Bittersohl Radiologische Diagnose des femoroazetabulären Impingements

\section{Zusammenfassung}

Beim femoroazetabulären Impingement (FAI) bewirkt ein anatomisches Missverhältnis zwischen proximalem Femur und Azetabulum eine frühzeitige Abnützung der Gelenkflächen. Um Symptome wie eingeschränkte Beweglichkeit und Schmerzen zu beheben, aber auch um dem degenerativen Prozess vorzubeugen oder ihn zu verlangsamen, ist häufig eine Operation notwendig. Dabei hängt das Resultat vom präoperativen Gelenkstatus ab - mit schlechten Ergebnissen bei bereits fortgeschrittener Hüftgelenkarthrose. Dies erklärt die Notwendigkeit einer akkuraten Diagnostik, um Frühstadien der Gelenkschädigung erkennen zu können. Die Diagnostik des FAl beinhaltet klinische Untersuchung, Röntgendiagnostik und Magnetresonanztomographie (MRT). Die Standardröntgen-radiologische Untersuchung beim FAl wird anhand von 2 Röntgenaufnahmen durchgeführt, der a.p.-Beckenaufnahme sowie einer seitlichen Aufnahme des proximalen Femurs wie z. B. der "lateralen cross-table"- oder der Lauenstein-Aufnahme. Hierbei müssen Positionskriterien eingehalten werden, um Verzerrungsartefakte auszuschließen. Die MRT-Bildgebung ermöglicht eine Untersuchung der Hüfte in 3 Ebenen und sollte zudem radial geplante Sequenzen für eine verbesserte Darstellung der randnahen Strukturen wie Labrum und peripherem Knorpel beinhalten. Die Verwendung von Kontrastmittel für ein direktes MRArthrogramm (MRA) hat sich insbesondere für die Darstellung von Labrumschäden als vorteilhaft erwiesen. Die Datenlage in Hinblick auf die Knorpelbildgebung ist noch unklar. Weiterentwicklungen der Techniken werden in naher Zukunft die Diagnostik der Hüfte verbessern können. Hierzu zählen u. a. biochemisch sensitive MRT-Anwendungen.

\section{Schlüsselwörter}

Femoroazetabuläres Impingement (FAl) . Hüfte $\cdot$ Röntgen $\cdot$ MRT $\cdot$ Diagnose

\section{Radiological diagnosis of femoroacetabular impingement}

\section{Abstract}

Femoroacetabular impingements (FAl) are due to an anatomical disproportion between the proximal femur and the acetabulum which causes premature wear of the joint surfaces. An operation is often necessary in order to relieve symptoms such as limited movement and pain as well as to prevent or slow down the degenerative process. The result is dependent on the preoperative status of the joint with poor results for advanced arthritis of the hip joint. This explains the necessity for an accurate diagnosis in order to recognize early stages of damage to the joint. The diagnosis of FAl includes clinical examination, $\mathrm{X}$-ray examination and magnetic resonance imaging (MRI). The standard X-radiological examination for FAl is carried out using two X-ray images, an anterior-posterior view of the pelvis and a lateral view of the proximal femur, such as the cross-table lateral or Lauenstein projections. It is necessary that positioning criteria are adhered to in order to avoid distortion artifacts. MRI permits an examination of the pelvis on three levels and should also include radial planned sequences for improved representation of peripheral structures, such as the labrum and peripheral cartilage. The use of contrast medium for a direct MR arthrogram has proved to be advantageous particularly for representation of labrum damage. The data with respect to cartilage imaging are still unclear. Further developments in technology, such as biochemical-sensitive MRI applications, will be able to improve the diagnosis of the pelvis in the near future.

\section{Keywords}

Femoroacetabular impingement (FAl) · Hip . $\mathrm{X}$-ray - Magnetic resonance imaging . Diagnosis 


\section{Leitthema: Hüftdiagnostik}
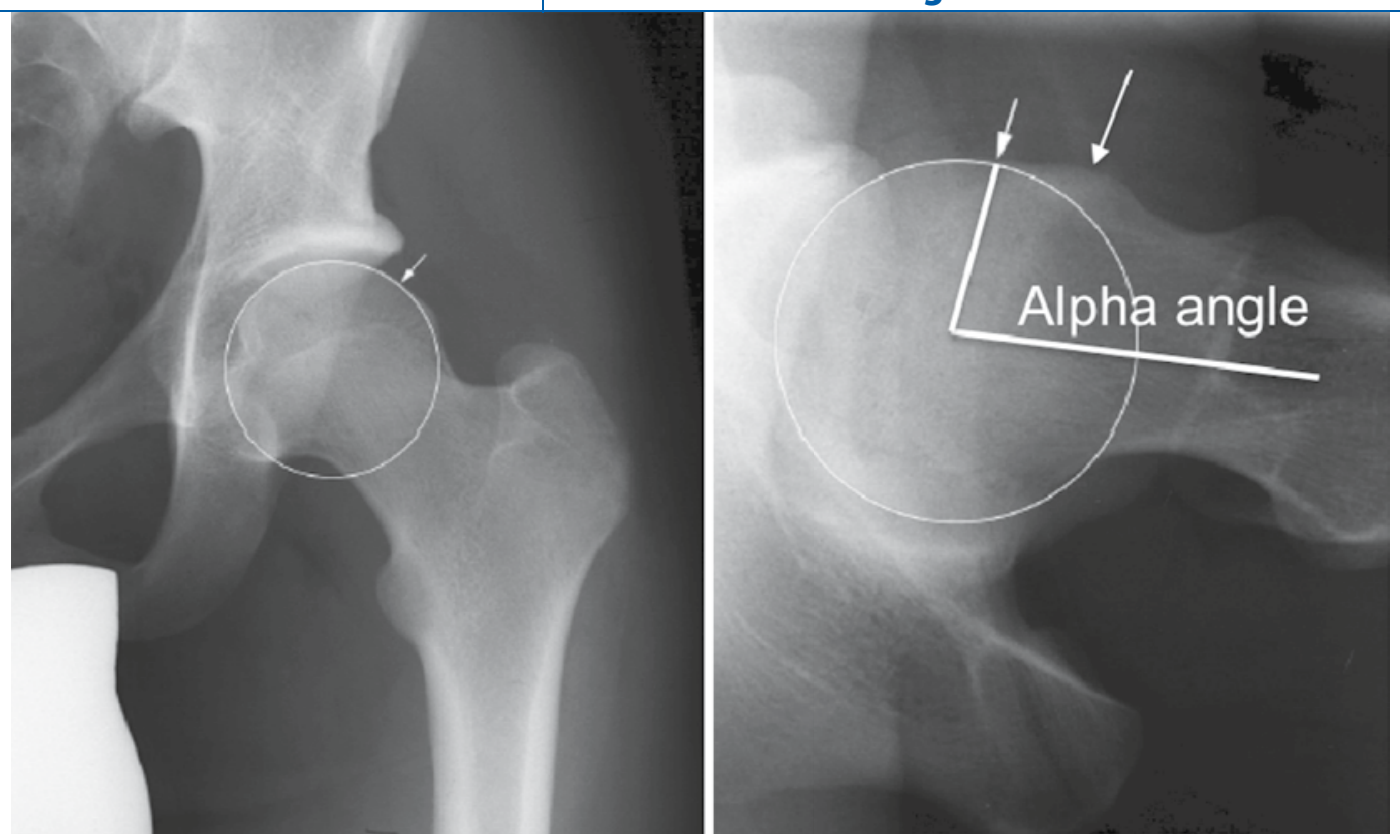

Abb. $1 \varangle$ Verminderte Taillierung (Offset) am KopfHals-Übergang bei einem Cam-FAl-Patienten. Es zeigt sich in der a.p.-Röntgenaufnahme eine leichte Aspherizität. Die axiale Aufnahme zeigt dagegen deutlich die Veränderung am KopfHals-Übergang (langer Pfeil). Bestimmung des aWinkels zur Quantifizierung der Veränderung
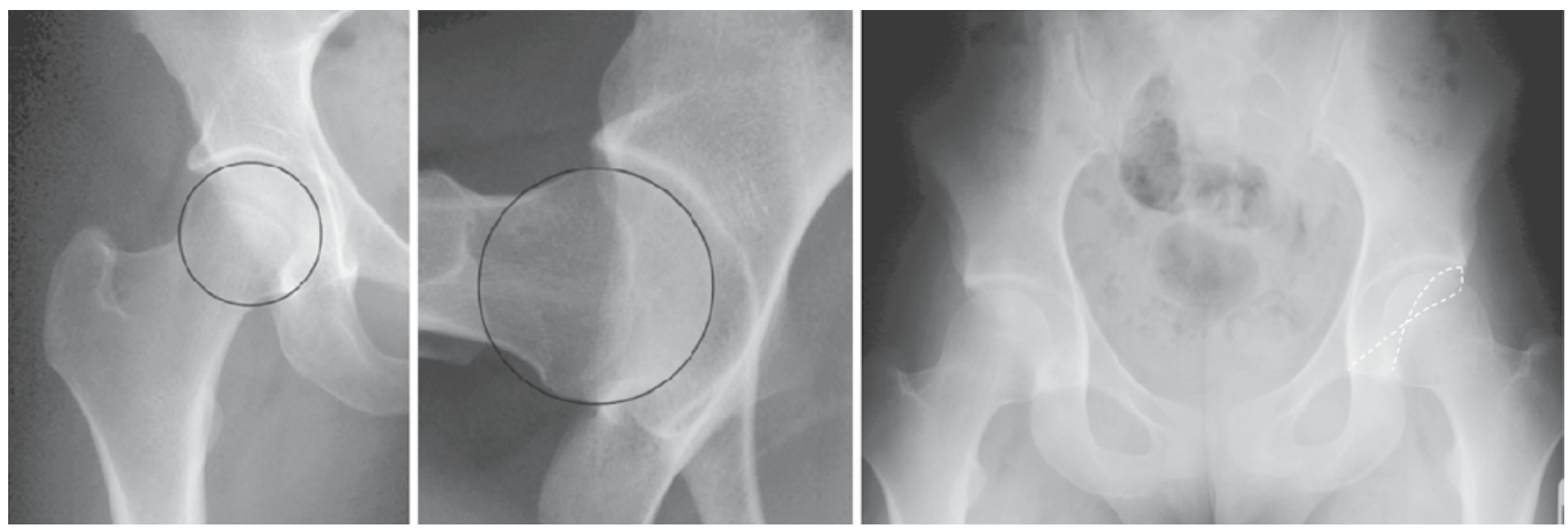

Abb. $2 \Delta$ Bestimmung azetabulärer Veränderungen mittels Röntgenuntersuchung. Links und Mitte: Coxa profunda mit pathologischer Mehrüberdachung im Hüftgelenk. Rechts: Bestimmung der azetabulären Version anhand der Stellung des vorderen zum hinteren Azetabulumrandes. Beachte das "cross over sign" als Zeichen einer azetabulären Retroversion

tomatischen Hüften wurden hierbei Off-

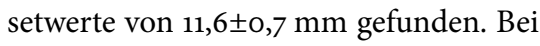
Cam-Impingement-Patienten findet sich ein vermindertes Offset $(7,2 \pm 0,7 \mathrm{~mm}[38])$ Für die klinische Anwendung können Offsetwerte $<10 \mathrm{~mm}$ als Hinweis für eine pathologische Verminderung der Taillierung zwischen Kopf und Schenkelhals angesehen werden [24]. Die Offsetratio ist der Quotient aus anteriorem Offset und Femurkopfdurchmesser. Bei asymptomatischen Hüften beträgt die mittlere Offsetratio $0,21 \pm 0,03 \mathrm{~mm}$, bei Cam-Impingement-Patienten $0,13 \pm 0,05 \mathrm{~mm}$ [24].

\section{Typische Röntgenradiologische Zeichen beim Pincer-Impingement}

Eine Coxa profunda, azetabuläre Protrusion und azetabuläre Retroversion sind typisch für ein Pincer-Impingement [24]. Folgende Parameter finden sich in der a.p.-Beckenaufnahme:

- Eine Coxa profunda liegt vor, wenn sich die Fossa acetabularis auf oder medial der Ilioischiallinie projiziert (• Abb. 2).

- Wenn der Femurkopf in der Projektion die Ilioischiallinie überschreitet, bezeichnet man das als azetabuläre Protrusion.
Beide Formen (Coxa profunda, azetabuläre Protrusion), die auf eine erhöhte Tiefe der Fossa acetabularis hindeuten, sind Indikatoren für eine generalisierte azetabuläre Mehrüberdachung. Diese kann mittels LCE- (Lateral-center-edge)Winkel [39] und femoralem Extrusionsindex [40] quantifiziert werden. Der LCEWinkel wird gebildet zwischen dem Lot durch das Femurkopfzentrum und der Tangente an den lateralen Azetabulumrand. Als normal gelten Werte zwischen 25 und $39^{\circ}$. Werte $<25^{\circ}$ weisen auf eine Dysplasie hin [1], während Werte $>39^{\circ}$ auf eine azetabuläre Mehrüberdachung deuten [29], obgleich diese Angaben nur als Indikatoren anzusehen sind [24]. Werte zwischen 25 und $39^{\circ}$ schließen eine Mehr- 
und/oder Minderüberdachung nicht aus und umgekehrt. Der femorale Extrusionsindex ist der prozentuale Anteil des nichtüberdachten Femurkopfs. Als Norm gelten Werte $<25 \%$ [41].

Eine lokale Mehrüberdachung als mögliche Ursache für das Pincer-Impingement kann anterior wie auch posterior bestehen. Physiologisch ist das Azetabulum antevertiert, d. h. der vordere Azetabulumrand liegt medial des Azetabulumhinterrandes. Dies spiegelt sich in der a.p.Beckenaufnahme wider. Bei der azetabulären Retroversion dagagen projiziert sich der vordere Azetabulumrand lateral des hinteren Azetabulumrandes [14, 15, 42]. Dies zeigt sich in der a.p.-Beckenaufnahme durch das so genannte"Cross-overZeichen”, welches das Kreuzen von Vorder- und Hinterrand ("8er"-Figur) im distalen Azetabulum zeigt (• Abb. 2). Eine prominente Hinterwand des Azetabulums als mögliche Ursache für ein posteriores Impingement kann mittels des so genannten Hinterwandzeichens evaluiert werden [24]. Das Hinterwandzeichen wird positiv gewertet, wenn sich der Hinterrand des Azetabulums lateral des Femurkopfzentrums projiziert. Eine prominente Hinterwand wird häufig auch bei einer generalisierten Mehrüberdachung festgestellt.

Wie oben erwähnt, findet sich beim Pincer-Impingement bei ca. 1/3 der Patienten eine Contre-coup-Läsion im posterioren Bereich $[4,10,26]$, hervorgerufen durch eine Hebelwirkung beim frontalen Anschlagen an den Azetabulumvorderrand. Diese Läsion, einhergehend mit einem Gelenkspaltverlust in dieser Region, kann mittels der lateralen Cross-table- und der "Faux-Profil"-Aufnahme ("Falsch-Profil-Aufnahme") dargestellt werden. Bei der von Lequesne u. Laredo [43] erstbeschriebenen Faux-Profil-Aufnahme steht der Patient mit der zu untersuchenden Hüfte am Röntgenschirm. Die Gegenseite wird soweit nach posterior zurückgenommen, dass das Becken mit einem Winkel von $65^{\circ}$ zum Röntgenschirm steht. Der Röntgenstrahl verläuft dagegen senkrecht zum Röntgenschirm durch das Becken. Mit dieser Projektion lassen sich neben den posterioren Hüftkopfkonturen auch der vorderer Gelenkspalt und insbesondere die anteriore Pfannenüberdachung gut darstellen.

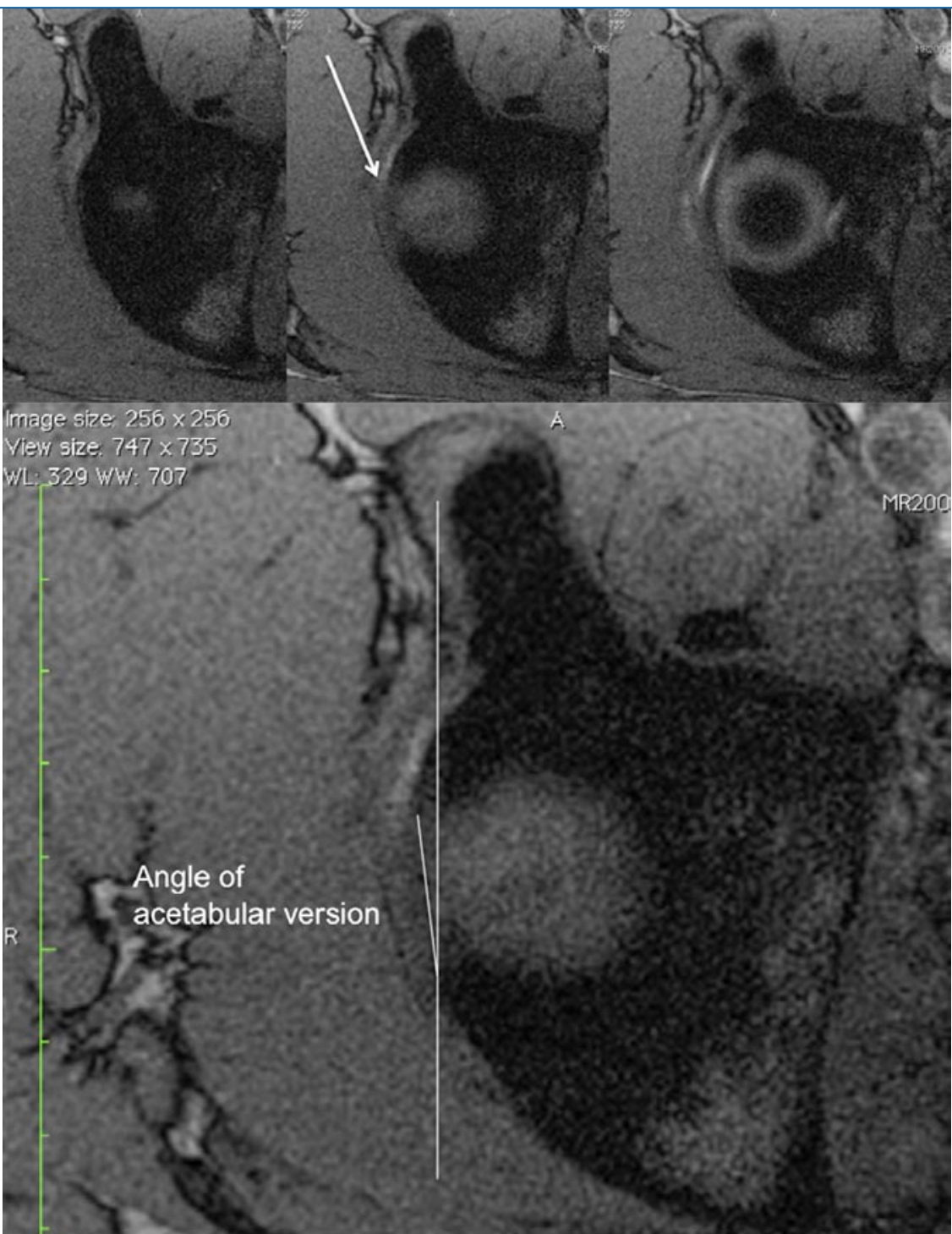

Abb. $3 \Delta$ Bestimmung der azetabulären Version im MRT mittels einer axial geplanten T1w-Sequenz durch das azetabuläre Dach. Obere Reihe: In den axialen Schichten zeigt sich die Öffnung des Azetabulums mit vorderem und hinterem Azetabulumrand (Pfeil). Untere Reihe: In dieser Ebene Bestimmung der azetabulären Version als Winkel zur Verbindungslinie zwischen vorderem und hinterem Rand

Ein typischer Befund (33\%) beim FAI sind die so genannten"herniation pits" $[44,45]$, die sich häufig im vorderen oberen Quadranten des Schenkelhalses befinden. Dabei handelt es sich um Knochenzysten, die sich im Röntgenbild als Signalauslöschung, umgeben von einem Sklerosesaum, darstellen [45].

Röntgenradiologisch wird der Gelenkstatus anhand der Arthrosegrade nach Tönnis klassifiziert [46]. Dies beinhaltet die Beurteilung der Gelenkspaltweite in der gewichttragenden Zone in der a.p.-Beckenaufnahme sowie den Nachweis subchondraler Sklerosierung, osteophytärer Anbauten und Geröllzysten.
Die genannten Untersuchungen im Röntgenbild sind unabdingbar zur Diagnose und FAI-Klassifikation. Jedoch sind die im Röntgenbild typischen Arthrosezeichen bereits Indikatoren für eine fortgeschrittene Gelenkdegeneration. Zudem lassen sich Strukturen wie Labrum und Knorpel nur indirekt beurteilen. Aufgrund der zweidimensionalen Limitierung können komplexere morphologische Gegebenheiten, wie z. B. eine bestimmte Femurkopf-Schenkelhals-Region mit verminderter Taillierung, durch Überprojektion übersehen werden [47]. Dies ist von Bedeutung, weil 


\section{Leitthema: Hüftdiagnostik}

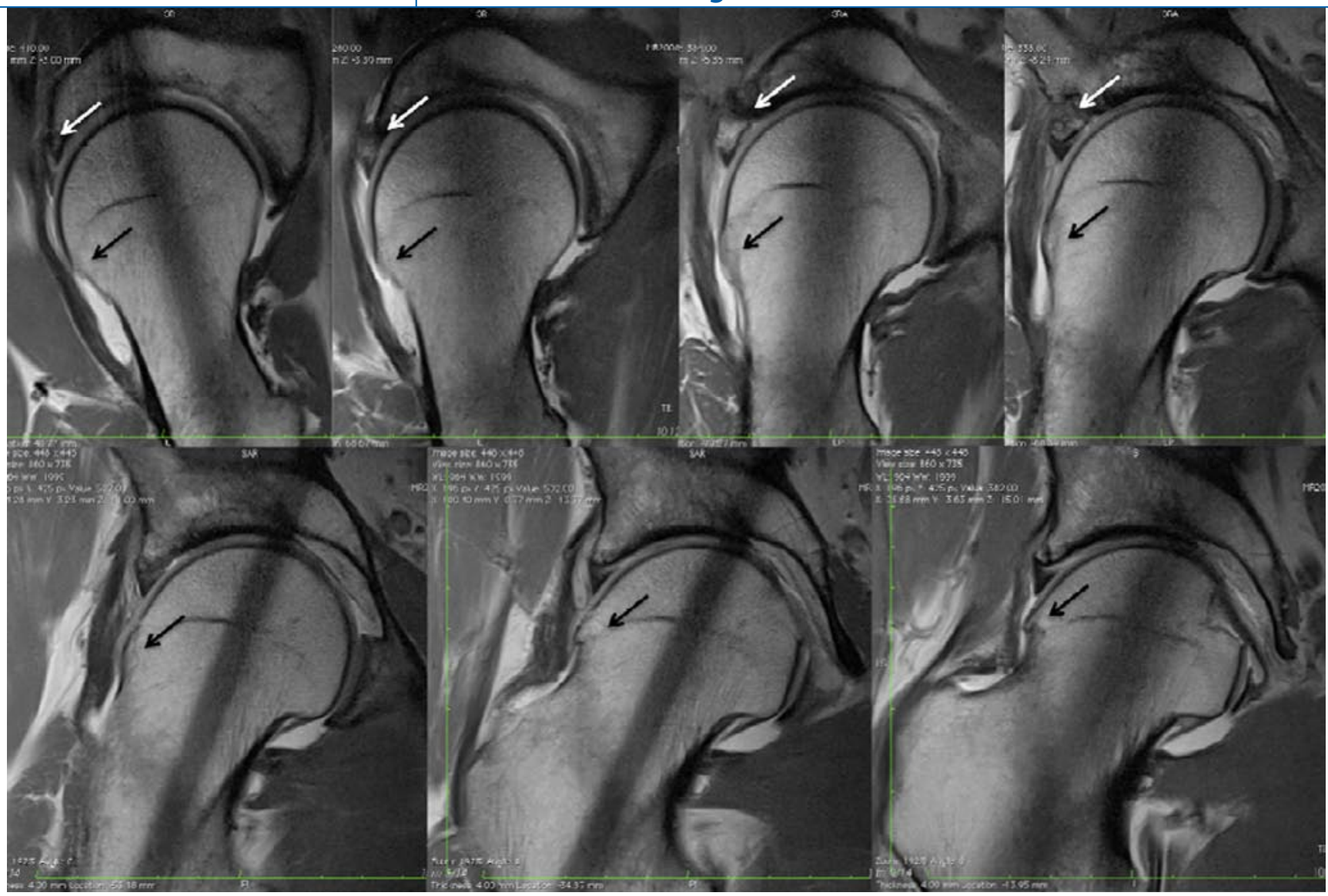

Abb. $4 \Delta$ Radiäre Schnittführung um den Femurhals (PD-gewichtete Turbospinecho- [PD-TSE-]Sequenz). Bestimmung der Femurmorphologie (schwarze Pfeile) mit Abnahme der Kopf-Hals-Taillierung von anterior nach superior. Diagnose der Labrumveränderungen mit orthogonalem Anschnitt des Labrums und genauer anatomischer Lokalisation (weiße Pfeile)

- in der Initialphase des FAI sich im Röntgenbild nicht die typischen Arthrosemerkmale zeigen,

- FAI-Patienten in unterschiedlichem Maße von einer Gelenkdegeneration - oft unabhängig vom klinischen Befund - betroffen sind,

- die Progression der Knorpeldegeneration unvorhersehbar ist und

- die Ergebnisse nach gelenkerhaltenden Operationen abhängig vom Grad der präoperativen Knorpelschädigung sind.

\section{Magnetresonanztomographie und Magnetresonanzarthrographie}

Die MRT ist ein Standardverfahren zur Beurteilung des Hüftgelenks. Der hohe Weichteilkontrast ermöglicht eine direkte Beurteilung von Labrum, Knorpel und anderen Gelenkstrukturen ohne den schädigenden Einfluss ionisierender Röntgenstrahlen [48]. Zudem handelt es sich um eine Tomographietechnik und somit können verschiedene Regionen des Gelenks separat evaluiert werden. Jedoch sind die Gegebenheiten in der Hüfte explizit. Die in allen Ebenen sphärisch gegenüberstehenden Gelenkflächen, die enge Nachbarschaft von Gelenkstrukturen wie Gelenkkapsel, Labrum, azetabulärer und femoraler Knorpel sowie die geringe Knorpeldicke erfordern hohe technische Voraussetzungen wie eine sehr hohe Bildauflösung und ein hohes Kontrastzu-Rauschen-Verhältnis (CNR, „,contrast to noise ratio").

Momentan hat sich die Zuhilfenahme von Kontrastmittel (unter Röntgenkontrolle und streng sterilen Bedingungen intraartikulär appliziert) für ein direktes MR-Arthrogramm durchgesetzt $[49,50]$, denn durch die Kontrastmittelfüllung in die natürlichen und auch pathologisch entstandenen Hohlräume und Spalten mit einhergehender Signalverstärkung erhöht es die Sensitivität und Genauigkeit der Diagnose möglicher Schäden. Zudem wird durch das Kontrastmittel die Gelenkkap- sel vom Labrum abgehoben und auch der Gelenkspalt etwas erweitert, was eine bessere Erkennung der unterschiedlichen Strukturen ermöglicht.

In der Routine werden MRT-Schnittbilder in allen 3 Ebenen (koronar, sagittal und axial) angefertigt, um die Morphologie und die Version des Azetabulums (• Abb. 3), Femurkopf-Hals-Offset und etwaige Knochen-, Knorpel- und Weichteilschäden akkurat diagnostizieren zu können. Zusätzlich hat sich die radiale Planung der Schnittebenen für die Beurteilung von Labrum und randnahem Knorpel als vorteilhaft erwiesen $[11,51,52$, 53], weil damit diese Regionen weitestgehend orthogonal angeschnitten und somit verzerrungsfrei dargestellt werden (- Abb. 4). Diese radialen Schnitte ermöglichen auch eine Rundumbetrachtung des Femurkopf-Hals-Übergangs. Mittels a-Winkel, Offset oder Offsetratio kann somit die Morphologie in diesem Bereich genau evaluiert werden, was das Risiko, subtile Anomalien z. B. durch 


\begin{abstract}
Abb. 5 - Bestimmung der Kopf-Hals-Taillierung mittels a-Winkel und Offsetratio $(r 2 / r 1)$. Der $a-W i n-$ kel beschreibt den Winkel zwischen einer Parallelen durch den Femurhals, die senkrecht zum Femurhalsdurchmesser und durch den Mittelpunkt des Femurkopfs verläuft, und einer Linie vom Mittelpunkt zu der Stelle, an der die Sphärizität des Femurkopfs zum Hals aufgehoben wird
\end{abstract}

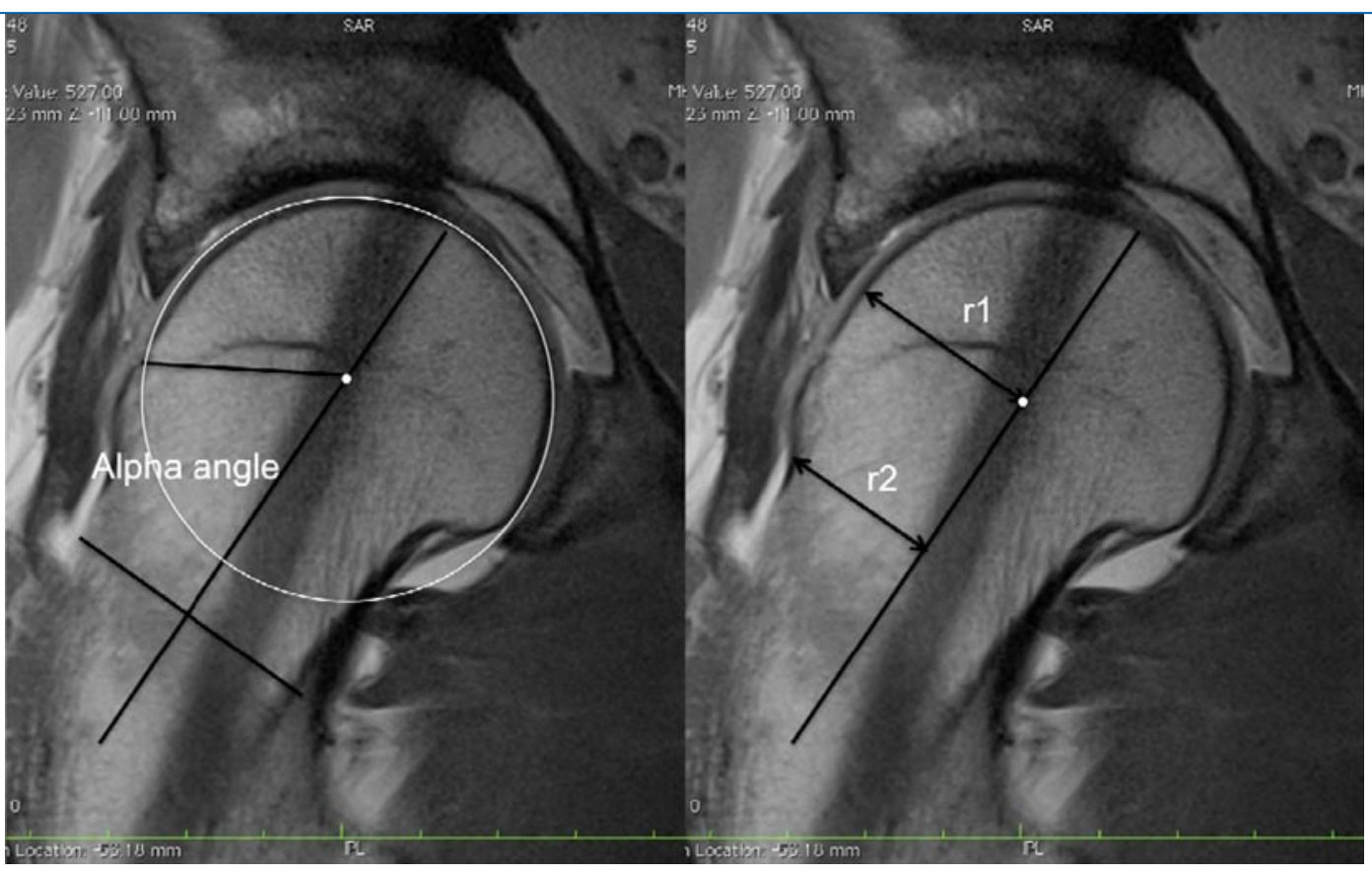

Überprojektion zu übersehen, vermindert (• Abb. 5, 6, 7). Dies ist von Bedeutung, da dadurch ein FAI besser erkannt, verstanden und mittels einer möglichen Operation, dem Ausmaß der Anomalie angepasst, entsprechend angegangen werden kann.

\section{Beurteilung des Labrums}

Zur Beurteilung des azetabulären Labrums werden MRT und MRA eingesetzt, wobei sich die MRA-Technik in Vergleichsstudien als vorteilhaft im Hinblick auf Sensitivität und Genauigkeit erwiesen hat $[52,54$, $56,57]$. Die publizierten Werte für die Sensitivität der MRA für die Erkennung von Labrumschäden reichen von $71-90 \%$. Jedoch wurde in den meisten Studien nur Schaden vs. kein Schaden unterschieden und diese Befunde verschiedenen Regionen wie anterior, superior und posterior zugeordnet.

Eine genauere Klassifizierung des Labrumschadens wurde von Czerny et al. [54] vorgeschlagen. Hierbei werden unterschieden:

- Stadium o (normales Labrum: homogen geringe Signalintensität, dreieckige Form, Spalt zwischen Labrum und Gelenkkapsel, durchgehende Verbindung von Labrum und Azetabulum),

- Stadium 1A (kontrastmittelerhöhte Signalintensität im Zentrum des Labrum, dreieckige Form, Spalt zwischen

Abb. $6 \triangleright$ Impingementzyste (weißer Pfeil) als indirektes Zeichen eines FAI



Labrum und Gelenkkapsel, durchgehende Verbindung von Labrum und Azetabulum),

- Stadium $1 \mathrm{~B}$ (wie $1 \mathrm{~A}$, jedoch mit Vergrößerung/Verdickung des Labrums),

- Stadium 2A (erhöhte Signalintensität im gesamten Labrum, dreieckige Form, Spalt zwischen Labrum und Gelenkkapsel, durchgehende Verbindung von Labrum und Azetabulum),

- Stadium 2B (wie 2A, jedoch mit Vergrößerung/Verdickung des Labrums und Aufhebung des Spalts zwischen Gelenkkapsel und Labrum),

- Stadium 3A (das dreieckige geformte Labrum ist abgelöst vom Azetabulum) und
- Stadium ${ }_{3}$ B (das Labrum ist verdickt und abgelöst vom Azetabulum).

\section{Beurteilung des Knorpels}

Im Gegensatz zur allgemein positiv bewerteten MRA-Technik bzgl. des Labrums ist die Datenlage hinsichtlich der Beurteilbarkeit des femoroazetabulären Knorpels kontrovers. Während James et al. [58] eine hohe diagnostische Wertigkeit (89\% korrekt klassifizierte Knorpelschäden bei Hüftdysplasiepatienten) mittels nativer, hochauflösender MRT-Technik mit Verwendung einer Oberflächenspule und protonengewichteter Fast-Spinechosequenz erzielen konnten, waren 


\section{Leitthema: Hüftdiagnostik}

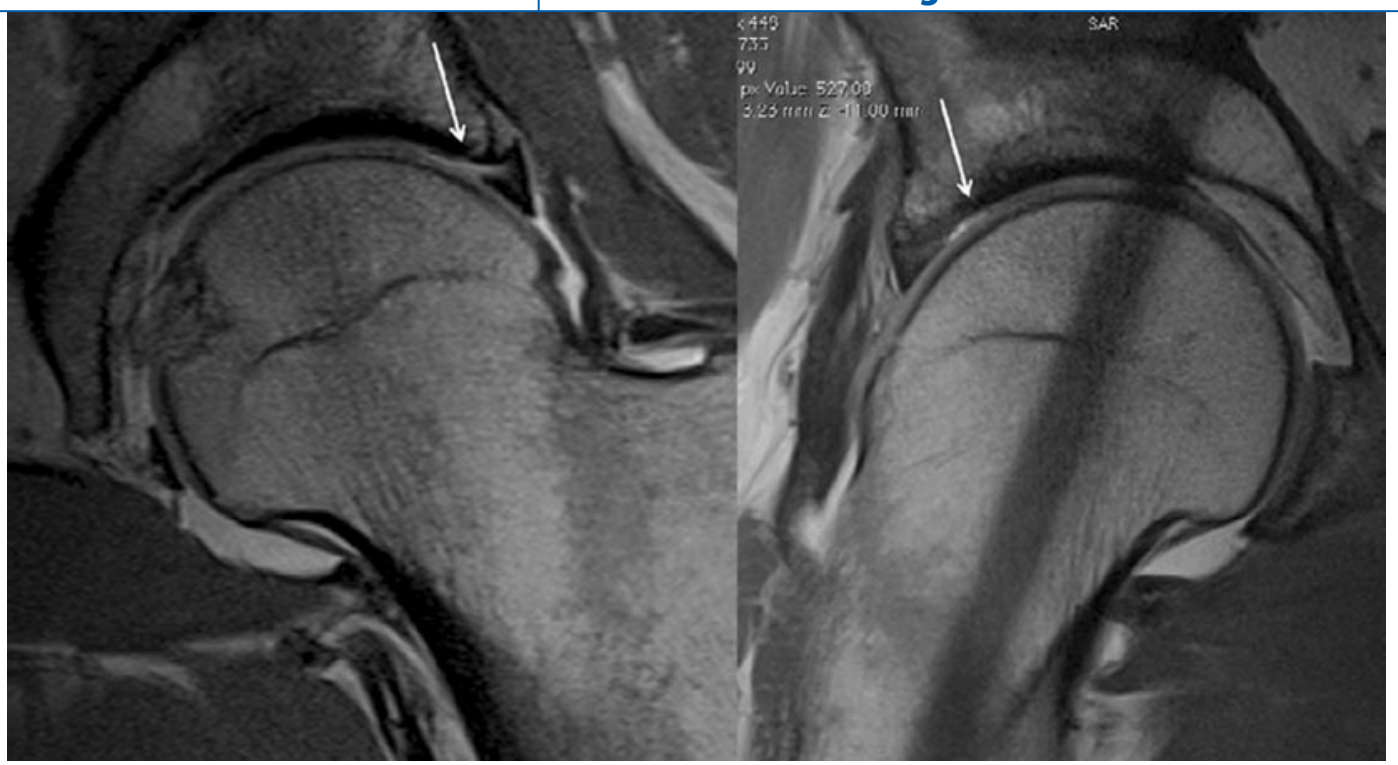

Abb. $7<$ Knorpelschaden am Azatabulumrand (weiBer Pfeil) an typischer Lokalisation bei Cam-Impingement. Koronale (linkes Bild) und radiäre Schnittführung an anterior-superiorer Position (rechtes Bild)

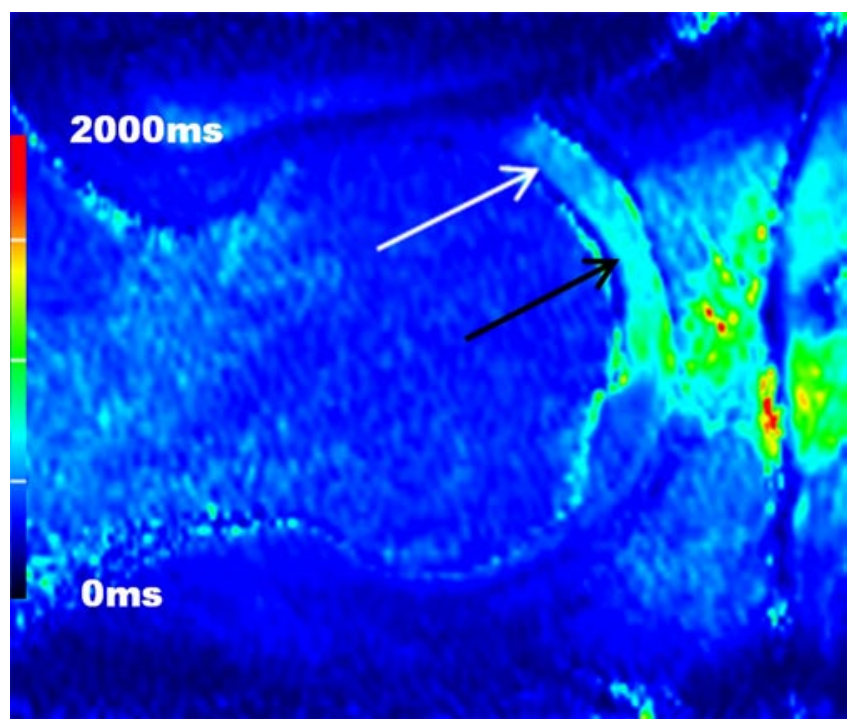

die Ergebnisse weiterer Studien weniger positiv. Bei diesen Arbeiten reichte die Sensitivität bei Knorpelschäden im Frühstadium von 47 [55] bis $49 / 67 \%$ (Auswerter 1/Auswerter 2; [59]), 58/62\% (Auswerter 1 /Auswerter 2) mittels 3 D-DESS-Technik (,double echo steady stat“") und TiwSpinechosequenz, und $69 / 81 \%$ (Auswerter 1/Auswerter 2) mittels protonengewichteter Sequenz mit Fettsättigung [6o]. Im Hinblick auf die sehr guten Resultate von James et al. [58] muss man erwähnen, dass zur Kontrolle der Knorpelbeurteilung mittels MRT eine Hüftarthroskopie durchgeführt wurde. Diese erlaubt keinen $360^{\circ}$-Rundblick und somit ist das Risiko erhöht, dass existierende Knorpelläsionen nicht registriert wurden. Zudem wurde ein grobes Einteilungsschema der
Abb. $8<$ dGEMRICMapping bei einem Cam-FAI-Patienten mit Verminderung der T1Relaxationszeit (weiBer Pfeil) nach Gadoliniumapplikation (T1Gd) am Azetabulumrand, was auf eine Verminderung des GAGGehalts an dieser Stelle hindeutet. Beachte die nicht verminderte GAG-Konzentration im Gelenkzentrum (schwarzer Pfeil)

\section{Ausblick}

Das Verständnis der beim FAI greifenden Pathomechanismen, die zu einer mechanisch induzierten Degeneration des Hüftgelenks führen, konnte in den letzten Jahren stetig erweitert werden. Dies wurde durch die klinische Relevanz als auch durch die Möglichkeit, den degenerativen Prozess mittels rechtzeitiger chirurgischer Intervention verlangsamen oder aufhalten zu können, weiter gefördert, was allerdings frühzeitig eine akkurate und praxisnahe Diagnostik erfordert.

Derzeit zeigt sich ein großes Interesse an biochemisch-sensitiven MRT-Techniken, die ergänzend zum Standardprotokoll angewendet werden können. Dies erklärt sich aus der Tatsache, dass initial degenerative Veränderungen wie ein Glykosaminoglykanverlust und Änderungen in der Kollagenfaserstruktur im Knorpelgewebe stattfinden, die noch nicht mit einer morphologischen Veränderung im Sinne einer Knorpelfaserung und/oder Knorpeldickenminderung einhergehen, jedoch mit diesen Techniken sensitiv aufgezeigt werden können. $\mathrm{Zu}$ diesen Techniken zählen u. a.

- diffusionsgewichtete Sequenzen [62],

- Tirho-Mapping [63],

- dGEMRIC [64],

- $\mathrm{T}_{2}$ - [65] und $\mathrm{T}_{2}{ }^{*}$-Mapping [66]. eine Vergleichsstudie zwischen einer nativen 3-T-MRT- und einer 1,5-T-MRAUntersuchung festgestellt werden konnte [61].
Beim Hüftgelenk wurden bisher aussichtsreiche Resultate mit dGEMRIC [67, 68] und T2-Mapping [59] erzielt. Wäh- 
rend das Tirho- und das dGEMRIC Verfahren den Verlust an Glykosaminoglykanen (GAG) im Knorpel detektieren (- Abb. 8), reagieren die $\mathrm{T} 2$ - und $\mathrm{T}_{2}{ }^{*}$ Mapping-Techniken auf die Veränderung der Integrität des Knorpelfasernetzwerks. Auch im Hinblick auf die FAI-Erforschung sind diese Techniken Grundlage weiterer Studien, deren Resultate in naher Zukunft zur Verfügung stehen werden.

\section{Korrespondenzadresse \\ Dr. T.C. Mamisch}

Abt. Orthopädische Chirurgie, Inselspital, Freiburgstraße, CH-3010 Bern

Schweiz

mamisch@bwh.harvard.edu

Interessenkonflikt. Der korrespondierende Autor gibt an, dass kein Interessenkonflikt besteht.

\section{Literatur (Auswahl)}

3. Harris WH (1986) Etiology of osteoarthritis of the hip. Clin Orthop Relat Res 213:20-33

4. Ganz R, Parvizi J, Beck M et al (2003) Femoroacetabular impingement: a cause for osteoarthritis of the hip. Clin Orthop Relat Res 417:112-120

5. Ganz R, Bamert P, Hausner P et al (1991) Cervicoacetabular impingement after femoral neck fracture. Unfallchirurg 94:172-175

6. Stulberg S, Cordell L, Harris W et al (1975) Unrecognized childhood hip disease: a major cause of idiopathic osteoarthitis of the hip. Proceedings of the Third Open Scientific Meeting of the Hip, pp 212-228

8. Resnick D (1976) The,tilt deformity' of the femoral head in osteoarthritis of the hip: a poor indicator of previous epiphysiolysis. Clin Radiol 27:355-363

9. Myers SR, Eijer H, Ganz R (1999) Anterior femoroacetabular impingement after periacetabular osteotomy. Clin Orthop Relat Res 363:93-99

10. Beck M, Kalhor M, Leunig M, Ganz R (2005) Hip morphology influences the pattern of damage to the acetabular cartilage: femoroacetabular impingement as a cause of early osteoarthritis of the hip. J Bone Joint Surg [Br] 87:1012-1018

11. Ito K, Minka MA 2nd, Leunig M et al (2001) Femoroacetabular impingement and the cam-effect. $A$ MRI-based quantitative anatomical study of the femoral head-neck offset. J Bone Joint Surg [Br] 83:171-176

12. Leunig M, Ganz R (2005) Femoroacetabular impingement. A common cause of hip complaints leading to arthrosis. Unfallchirurg 108:9-10, 12-17

13. Notzli HP, Wyss TF, Stoecklin CH et al (2002) The contour of the femoral head-neck junction as a predictor for the risk of anterior impingement. J Bone Joint Surg [Br] 84:556-560

15. Siebenrock KA, Schoeniger R, Ganz R (2003) Anterior femoro-acetabular impingement due to acetabular retroversion. Treatment with periacetabular osteotomy. J Bone Joint Surg [Am] 85-A:278-286

16. Wagner S, Hofstetter W, Chiquet M et al (2003) Early osteoarthritic changes of human femoral head cartilage subsequent to femoro-acetabular impingement. Osteoarthritis Cartil 11:508-518
17. Siebenrock KA, Wahab KH, Werlen S et al (2004) Abnormal extension of the femoral head epiphysis as a cause of cam impingement. Clin Orthop Relat Res 54-60

18. Eijer H, Myers SR, Ganz R (2001) Anterior femoroacetabular impingement after femoral neck fractures. J Orthop Trauma 15:475-481

19. Goodman DA, Feighan JE, Smith AD et al (1997) Subclinical slipped capital femoral epiphysis. Relationship to osteoarthrosis of the hip. J Bone Joint Surg [Am] 79:1489-1497

20. Leunig M, Casillas MM, Hamlet M et al (2000) Slipped capital femoral epiphysis: early mechanical damage to the acetabular cartilage by a prominent femoral metaphysis. Acta Orthop Scand 71:370-375

23. Giori NJ, Trousdale RT (2003) Acetabular retroversion is associated with osteoarthritis of the hip. Clin Orthop Relat Res 417:263-269

28. Beck M, Leunig M, Parvizi J et al (2004) Anterior femoroacetabular impingement: part II. Midterm results of surgical treatment. Clin Orthop Relat Res 418:67-73

31. Ganz R, Gill TJ, Gautier E et al (2001) Surgical dislocation of the adult hip a technique with full access to the femoral head and acetabulum without the risk of avascular necrosis. J Bone Joint Surg [Br] 83:1119-1124

35. Siebenrock KA, Kalbermatten DF, Ganz R (2003) Effect of pelvic tilt on acetabular retroversion: a study of pelves from cadavers. Clin Orthop Relat Res 407:241-248

38. Eijer H, Leunig M, Mahomed M, Ganz R (2001) Cross-table lateral radiograph for screening of anterior femoral head-neck offset in patients with femoro-acetabular impingement. Hip Int 11:37-41

44. Leunig M, Beck M, Kalhor M et al (2005) Fibrocystic changes at anterosuperior femoral neck: prevalence in hips with femoroacetabular impingement. Radiology 236:237-246

45. Pitt MJ, Graham AR, Shipman JH, Birkby W (1982) Herniation pit of the femoral neck. AJR Am J Roentgenol 138:1115-1121

47. Dudda M, Albers C, Mamisch TC et al (2008) Do normal radiographs exclude asphericity of the femoral head-neck junction? Clin Orthop Relat Res 467:651-659

48. Mamisch TC, Bittersohl B, Hughes T et al (2008) Magnetic resonance imaging of the hip at 3 tesla: clinical value in femoroacetabular impingement of the hip and current concepts. Semin Musculoskelet Radiol 12:212-222

49. Pfirrmann CW, Mengiardi B, Dora C et al (2006) Cam and pincer femoroacetabular impingement: characteristic MR arthrographic findings in 50 patients. Radiology 240:778-785

51. Kubo T, Horii M, Harada Y et al (1999) Radial-sequence magnetic resonance imaging in evaluation of acetabular labrum. J Orthop Sci 4:328-332

52. Leunig M, Werlen S, Ungersbock A et al (1997) Evaluation of the acetabular labrum by MR arthrography. J Bone Joint Surg [Br] 79:230-234

53. Locher S, Werlen S, Leunig M, Ganz R (2002) MR arthrography with radial sequences for visualization of early hip pathology not visible on plain radiographs. Z Orthop Ihre Grenzgeb 140:52-57

55. Keeney JA, Peelle MW, Jackson J et al (2004) Magnetic resonance arthrography versus arthroscopy in the evaluation of articular hip pathology. Clin Orthop Relat Res 429:163-169
61. Sundberg TP, Toomayan GA, Major NM (2006) Evaluation of the acetabular labrum at 3.0-T MR imaging compared with 1.5-T MR arthrography: preliminary experience. Radiology 238:706-711

64. Burstein D, Velyvis J, Scott KT et al (2001) Protocol issues for delayed Gd(DTPA)(2-)-enhanced MRI (dGEMRIC) for clinical evaluation of articular cartilage. Magn Reson Med 45:36-41

68. Kim YJ, Jaramillo D, Millis MB et al (2003) Assessment of early osteoarthritis in hip dysplasia with delayed gadolinium-enhanced magnetic resonance imaging of cartilage. J Bone Joint Surg [Am] 85-A:1987-1992

Das vollständige Literaturverzeichnis ...

... finden Sie in der html-Version dieses Beitrags im Online-Archiv auf der Zeitschriftenhomepage www.DerRadiologe.de 